\title{
The discovery and study of the optical counterparts of the transient X-ray pulsars RX J0052.1-7319 and XTE J0111.2-7317 in the SMC ${ }^{\star}$
}

\author{
S. Covino ${ }^{1}$, I. Negueruela ${ }^{2}$, S. Campana ${ }^{1}$, G. L. Israel ${ }^{3, \star \star}$, V. F. Polcaro ${ }^{4}$, L. Stella ${ }^{3, \star \star}$, and F. Verrecchia ${ }^{5}$ \\ 1 Osservatorio Astronomico di Brera, Via Bianchi 46, 23807 Merate (LC), Italy \\ 2 Observatoire de Strasbourg, 11 rue de l'Université, 67000 Strasbourg, France \\ 3 Osservatorio Astronomico di Roma, Via Frascati 33, 00040 Monteporzio Catone, Roma, Italy \\ 4 Istituto di Astrofisica Spaziale, Via Fosso del Cavaliere, 00133 Roma, Italy \\ 5 Università "La Sapienza", Dipartimento di Fisica, Piazzale A. Moro 5, 00185, Roma, Italy
}

Received 3 April 2001 / Accepted 23 May 2001

\begin{abstract}
We report on the discovery and confirmation of the optical counterparts of the two transient X-ray pulsars, RX J0052.1-7319 and XTE J0111.2-7317. In the narrow ( $3^{\prime \prime}$ radius) X-ray error circle of RX J0052.17319 we found a $V=14.6(B-V=0.1)$ 09.5IIIe (a classification as a B0Ve star is also possible, since the luminosity class depends on the uncertainty on the adopted reddening). Medium resolution spectra for this object show Balmer lines in emission with an equivalent width of $\mathrm{H} \alpha=-12 \div-16 \AA$. In the $\mathrm{X}$-ray error box of XTE J0111.2-7317 we found a relatively bright object $(V=15.4, B-V=0.06)$ which has been classified as a B0.5-B1Ve star and that was later confirmed by Coe et al. (2000) as the most plausible counterpart for XTE J0111.2-7317. Also in this case we easily detect Balmer emission lines with an $\mathrm{H} \alpha$ equivalent width of about $-21 \AA$. There is also evidence for the presence of a surrounding nebula, possibly a supernova remnant. A further bright B0Ve star was found just outside the X-ray error circle of XTE J0111.2-7317. We discuss the implication of these results in the light of the current knowledge of Be/X-ray binary systems in the Magellanic Clouds and within our Galaxy.
\end{abstract}

Key words. pulsars: individual (RX J0052.1-7319, XTE J0111.2-7317) - stars: neutron - X-ray: stars

\section{Introduction}

X-ray pulsars in High Mass X-ray Binaries (HMXBs) consist of an accreting neutron star orbiting a (super)giant or a main-sequence Be-type companion star. The greater part of known neutron stars in Supergiant XBs (from now on SXBs) show permanently high X-ray fluxes driven by accretion of a roughly spherical dense wind from the massive companion (which may be enhanced by Roche lobe overflow). In contrast, the neutron stars in BeXBs often exhibit transient $\mathrm{X}$-ray outbursts which may occur periodically at periastron (type I) or when the companion star undergoes a mass loss episode from the equatorial regions due to its high rotational velocity (up to $\sim 75 \%$ of the break-up velocity; type II). In BeXBs, the primary star is an early type star in the range $10-20 M_{\odot}$ of luminosity

Send offprint requests to: S. Covino, e-mail: covino@merate.mi.astro.it

* Based on observations carried out at ESO, La Silla, Chile (62.H-0513, 63.H-0294(B) and 64.H-0059(A)).

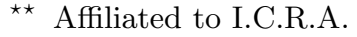

class III to V, which often displays Balmer lines, but also He I and metallic lines, in emission. Due to these lines, Be stars are difficult to classify. Moreover, since BeXBs are generally first detected as X-ray sources with positional accuracies which can be as poor as a few arcminutes, the optical/IR follow-up observations and the detection of the possible counterpart is often a difficult task. In addition, the BeXB concentration on the Galactic Plane and in the Magellanic Clouds (MCs) results in a further decrease in the chances of detecting them because of crowding and source confusion. So far only about $\sim 20$ optical counterparts of BeXBs have been discovered out of the $>100$ known and $10^{4}-10^{5}$ expected Be/X-ray pulsars (Nelson et al. 1993 and 1995). Detailed discussions on the nature and properties of these systems have been recently reported by several authors (Negueruela 1998; Coe 2000; Negueruela \& Okazaki 2000).

In this paper we report on the results of optical observations aimed at searching for the counterpart of two transient X-ray pulsars: RX J0052.1-7319 and XTE J0111.27317. Both systems are hosted by the Small Magellanic 
Table 1. Record of the observations.

\begin{tabular}{|c|c|c|c|c|c|c|c|c|c|}
\hline Date & Telescope & Field & Filter & $\begin{array}{c}\text { Grism/grating } \\
\text { ESO \# }\end{array}$ & $\begin{array}{c}\text { Exposure } \\
(\mathrm{sec})\end{array}$ & $\begin{array}{c}\text { Slit } \\
(\operatorname{arcsec})\end{array}$ & $\begin{array}{c}\text { Range } \\
(\AA) \\
\end{array}$ & $\begin{array}{c}\text { Resolution } \\
(\AA)\end{array}$ & $\begin{array}{c}\text { Seeing } \\
(\operatorname{arcsec}) \\
\end{array}$ \\
\hline 1999 Jan. 18 & $1.5 \mathrm{~m}$ Danish & RX J0052.1-7319 & $V$ & & 200 & & & & 2.5 \\
\hline 1999 Jan. 18 & $1.5 \mathrm{~m}$ Danish & RX J0052.1-7319 & $R$ & & 200 & & & & 1.5 \\
\hline 1999 Jan. 18 & $1.5 \mathrm{~m}$ Danish & XTE J0111.2-7317 & $V$ & & 200 & & & & 1.5 \\
\hline 1999 Jan. 18 & $1.5 \mathrm{~m}$ Danish & XTE J0111.2-7317 & $R$ & & 150 & & & & 2.0 \\
\hline 1999 Jan. 19 & $1.5 \mathrm{~m}$ Danish & RX J0052.1-7319 & $B$ & & 200 & & & & 3.0 \\
\hline 1999 Jan. 18 & $1.5 \mathrm{~m}$ Danish & XTE J0111.2-7317 & $B$ & & 200 & & & & 3.5 \\
\hline 1999 Jan. 19 & $1.5 \mathrm{~m}$ Danish & RX J0052.1-7319 & & 4 & 1000 & 2.0 & $3500-9000$ & 15 & 1.7 \\
\hline 1999 Jan. 19 & $1.5 \mathrm{~m}$ Danish & XTE J0111.2-7317 & & 4 & 1000 & 2.0 & $3500-9000$ & 15 & 1.8 \\
\hline 1999 Jan. 20 & $1.5 \mathrm{~m}$ Danish & RX J0052.1-7319 & & 8 & 2000 & 1.5 & $5800-8300$ & 5 & 1.7 \\
\hline 1999 Jan. 20 & $1.5 \mathrm{~m}$ Danish & XTE J0111.2-7317 & & 8 & 2000 & 2.0 & $5800-8300$ & 6 & 1.6 \\
\hline 1999 Sep. 14 & $3.6 \mathrm{~m} \mathrm{ESO}$ & XTE J0111.2-7317 & & 7 & 900 & 1.0 & $3300-5200$ & 6 & 1.0 \\
\hline 1999 Sep. 14 & $3.6 \mathrm{~m} \mathrm{ESO}$ & XTE J0111.2-7317 & & 9 & 900 & 1.0 & $4700-6800$ & 6 & 1.0 \\
\hline 1999 Sep. 15 & $3.6 \mathrm{~m} \mathrm{ESO}$ & RX J0052.1-7319 & & 7 & 2400 & 1.0 & $3300-5200$ & 6 & 1.1 \\
\hline 1999 Sep. 15 & $3.6 \mathrm{~m} \mathrm{ESO}$ & RX J0052.1-7319 & & 9 & 2400 & 1.0 & $4700-6800$ & 6 & 1.1 \\
\hline 1999 Nov. 2 & $1.5 \mathrm{~m} \mathrm{ESO}$ & XTE J0111.2-7317 & & 33 & 1500 & 2.0 & $3500-6000$ & 3 & 1.5 \\
\hline
\end{tabular}

Cloud (SMC) which harbors a large number of these systems (Yokogawa et al. 2000a; Mereghetti 2001).

The paper is organized as follows: in Sect. 2 we describe the techniques we have applied for the observations and the data reduction and in Sect. 3 we discuss the result of the observations and derive physical parameters for the proposed optical counterparts of RX J0052.1-7319 and XTE J0111.2-7317. The detailed spectral classification of the observed stars are also provided.

\section{Observations}

The search for the optical counterparts in the fields of the X-ray sources RX J0052-7319 and XTE J0111.2-7317 was carried out by means of several techniques and instruments. Photometry was performed in order to study the stellar field main properties and to select objects with colors and magnitudes expected for the counterparts of a BeXB in the SMC. Spectroscopy of the optical counterpart candidates was carried out to infer the physical parameters (i.e. spectral classification) and to look for signatures of activity to be correlated with the $\mathrm{X}$-ray emission.

Multicolor photometry ( $B, V$, and $R$ bands) was carried out for each field with the $1.5 \mathrm{~m}$ Danish telescope of the ESO at La Silla (Chile). Instrumental magnitudes for each stellar object in the images were derived with aperture and profile-fitting photometry by means of the DAOPHOT II package (Stetson 1987). Low and medium resolution spectroscopy was also performed at the $1.5 \mathrm{~m}$ Danish and $3.6 \mathrm{~m}$ telescopes of the ESO at La Silla for a number of selected bright objects in the $\mathrm{X}$-ray error circle. When the observing conditions were adequate we also performed absolute calibration both for photometry and spectroscopy. Spectroscopic data were analyzed by the "long" context in the ESO-MIDAS package (96NOV and later versions). Medium resolution spectroscopy of XTE J0111.2-7317 was also obtained with the ESO $1.5 \mathrm{~m}$ telescope. These observations were reduced with the FigARo package (Shortridge et al. 1997) of the Starlink suite. The $S / N$ of the spectra obtained at the
$3.6 \mathrm{~m}$ was always of the order of 150 (or better) while those obtained at the ESO $1.5 \mathrm{~m}$ had a $S / N$ around 50 . The spectral features discussed in the text were present in at least two spectra or clearly above the noise level.

A full record of the optical observations is reported in Table 1.

\section{Discussion}

\section{1. $R X J 0052.1-7319$}

The X-ray transient RX J0052.1-7319 was discovered by Lamb et al. (1999) with the analysis of ROSAT HRI and BATSE data. The object showed a period of $15.3 \mathrm{~s}$ (Kahabka 1998, 1999; Sasaki et al. 2000; for PSPC data analysis see also Haberl et al. 2000) and a flux in the 0.1$2 \mathrm{keV}$ band of $2.6 \times 10^{-11} \mathrm{erg} \mathrm{s}^{-1} \mathrm{~cm}^{-2}$.

We devoted particular care to the definition of the $\mathrm{X}-$ ray error circles of this source. The archival ROSAT HRI images, available for RX J0052-7319, were analyzed using both a sliding cell and a wavelet transform-based algorithm (Lazzati et al. 1999; Campana et al. 1999). These techniques, already applied by us to the ROSAT HRI fields of several X-ray sources (Israel et al. 1999a; Israel et al. 2000a; Covino et al. 2000; Israel et al. 2000b; Israel et al. 2000c; Mereghetti et al. 2001), allowed us to infer a positional accuracy in the $5 \div 10^{\prime \prime}$ range. In particular RX J0052.1-7319 was observed three times in the BMWHRI source catalog (see Table 2 ). The source is highly variable with a $\gtrsim 1,000$ variation in the count rate. The source is serendipituously detected at relatively large off-axis angles $\left(>8^{\prime}\right)$ affecting the source positioning. For each field we are able to perform a boresight correction, registering the $\mathrm{X}$-ray source positions to an optical frame. In the case of the shortest observation, we did not find $\mathrm{X}$-ray sources suitable for the boresight. For the remaining two observations we used for the boresight correction 3 and 1 sources, respectively. Boresight errors are summed up in quadrature with the source location error (see Table 2). We then combined the source positions obtained in the three observations to derive the final position: $\mathrm{RA}=00^{\mathrm{h}} 52^{\mathrm{m}} 13.27$, 
Table 2. Source characteristics of RX J0052.1-7319 in the BMW-HRI catalog.

\begin{tabular}{ccccccc}
\hline $\begin{array}{c}\text { Observation } \\
\text { (ROR) }\end{array}$ & RA (J2000) & DEC (J2000) & $\begin{array}{c}\text { Error } \\
\left({ }^{\prime \prime}, 90 \%\right)\end{array}$ & $\begin{array}{c}\text { Off-axis } \\
(\text { armin })\end{array}$ & $\begin{array}{c}\text { Exp. time } \\
(\mathrm{s})\end{array}$ & $\begin{array}{c}\text { Count rate } \\
\left(\mathrm{c} \mathrm{s}^{-1}\right)\end{array}$ \\
\hline rh300513n00 & 005212.60 & -731919.7 & 10.00 & 20.9 & 1582.4 & 0.9979 \\
rh600811n00 & 005214.41 & -731919.3 & 2.07 & 8.4 & 27395.0 & 0.0009 \\
rh600812a01 & 005213.10 & -731919.9 & 2.53 & 16.7 & 17064.5 & 0.6863 \\
\hline
\end{tabular}

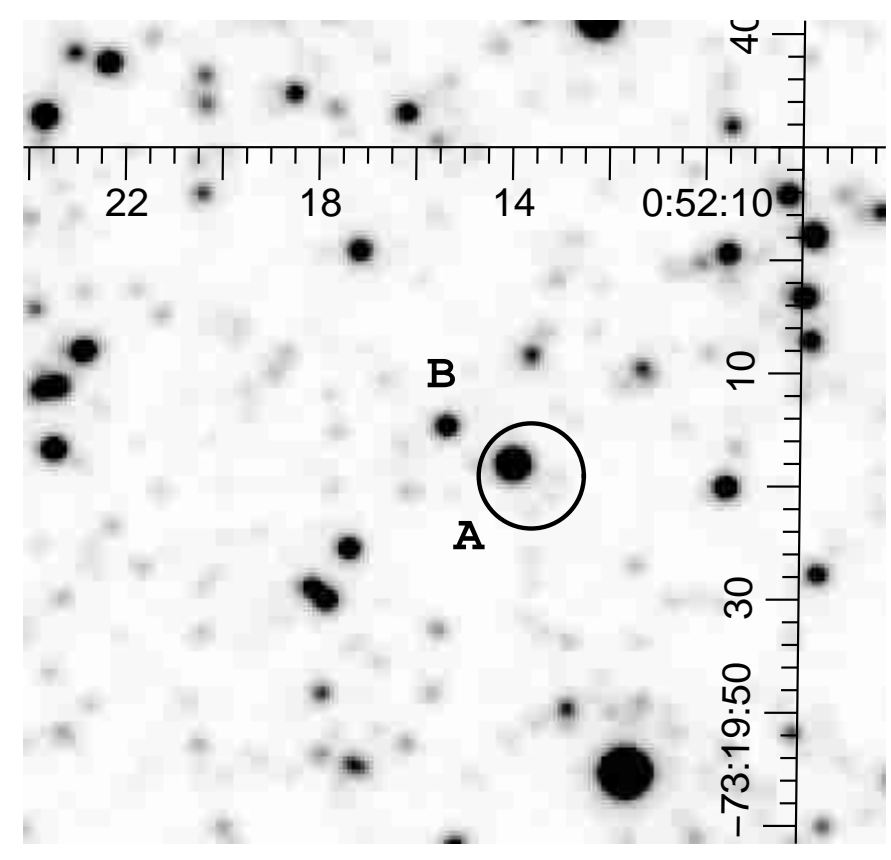

Fig. 1. 1.5 m ESO Danish telescope RX J0052-7319 $R$ image. The $\mathrm{X}$-ray error circle is also shown with the optical counterpart labeled as A. A nearby object is labeled as B.

DEC $=-73^{\circ} 19^{\prime} 19.5^{\prime \prime}(\mathrm{J} 2000)$. The $90 \%$ error circle radius amounts to $3.3^{\prime \prime}$.

An optical counterpart for this source was proposed (Israel et al. 1999b), a B-type star with $R=14.54 \pm 0.03$ and $V-R=+0.08 \pm 0.04$, located at coordinates (J2000, estimated uncertainty $\left.1^{\prime \prime}\right) \mathrm{RA}=00^{\mathrm{h}} 52^{\mathrm{m}} 13.9^{\mathrm{s}} .0$, DEC $=$ $-73^{\circ} 19^{\prime} 19^{\prime \prime}$. In Fig. 1 a $R$ frame for the RX J0052.1-7319 field is shown, together with the $\mathrm{X}$-ray error-circle. The proposed counterpart is the object labeled as A. This star was also identified in the OGLE photometric database (labeled as SMC_SC6 99923, Udalsky et al. 1999) as a longterm variable star with quasiperiodic light variation of amplitude $0.13 \mathrm{mag}$ in the $I$ band. This optical object is within the X-ray error circle of RX J0052.1-7319, lying at $2.8^{\prime \prime}$ from the best $\mathrm{X}$-ray position.

A $1000 \mathrm{~s}$ optical spectrum at the $1.5 \mathrm{~m}$ Danish telescope of the ESO at La Silla (Fig. 2) for object A shows $\mathrm{H} \alpha$ and $\mathrm{H} \beta$ emission ( $E W \sim-12$ and $-3 \AA$, respectively), supporting the Be nature of the star. Star B, just outside the $\mathrm{X}$-ray error circle, does not show any emission line. Its spectrum is compatible with being a moderately bright young SMC star somewhat later than object A.

The spectrum of the optical counterpart of RX J00527319 in the classification region was also studied with the $3.6 \mathrm{~m}$ ESO telescope and it is displayed in Fig. 3, together

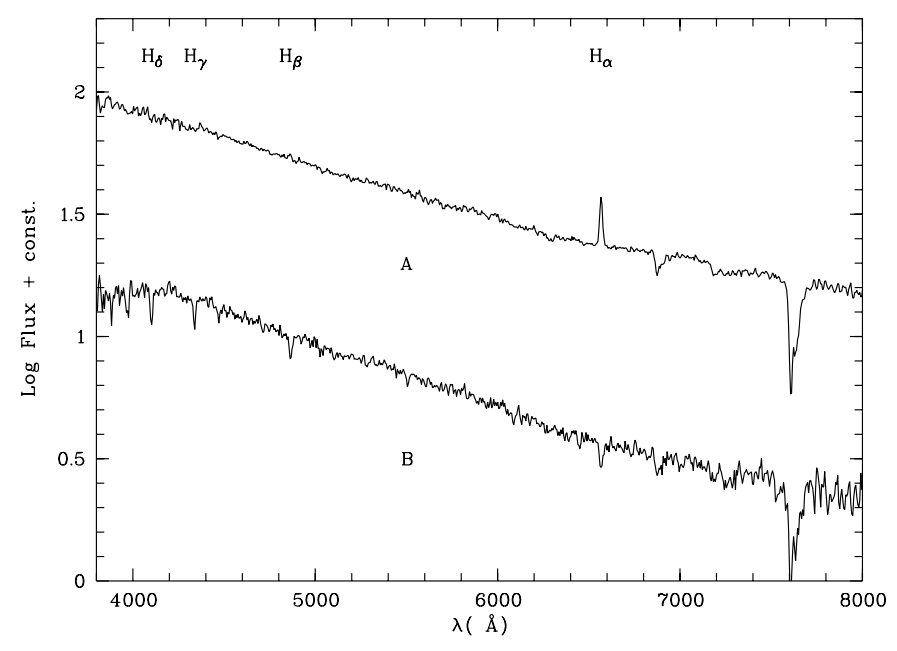

Fig. 2. 1.5 m ESO Danish telescope RX J0052-7319 spectrum for objects A and B. Position of the main hydrogen lines is also shown.

with that of the B0V MK standard $\nu$ Ori. $\mathrm{H} \beta$ and $\mathrm{H} \gamma$ are in emission and display asymmetric profiles. Some of the weaker He I lines are not visible, presumably filled in by emission. All the lines in the spectrum are very shallow and broad, which is typical of a Be/X-ray binary counterpart. The presence of weak He II lines places the object close to B0.

If the object is on the main sequence, the presence of weak HeII $\lambda 4200 \AA$ and the condition He II $\lambda 4541 \AA>$ Si III $\lambda 4552 \AA$ give a spectral type B0Ve. For this spectral type, an intrinsic $(B-V)_{0}=-0.26$ is expected (Wegner 1994). Therefore, the measured $(B-V)=$ 0.12 implies $E(B-V)=0.38$. Such reddening is rather high for a source in the SMC and would suggest that there is an important local contribution to the reddening. It is difficult to quantify if this could be due to localized absorption or to circumstellar reddening due to emission from the disc. The relationships of Fabregat \& Reglero (1990) would indicate for an $\mathrm{H} \alpha E W=-16 \AA$ the circumstellar contribution should only be $E^{\mathrm{cs}}(B-V) \simeq 0.04$, but this relationship is only valid for isolated Be stars and the envelopes of $\mathrm{Be} / \mathrm{X}$-ray binaries are known to be denser than those of isolated stars (Zamanov et al. 2001) - therefore the circumstellar contribution to the reddening could be higher.

The lower limit $E^{\mathrm{cs}}(B-V) \simeq 0.04$ would result in $M_{V}=-5.18 \pm 0.15$, using the distance modulus $(M-m)=18.75 \pm 0.07$ (Udalski 2000). This intrinsic magnitude is far too bright for a main-sequence object and would suggest that the star is a giant. Alternatively, 


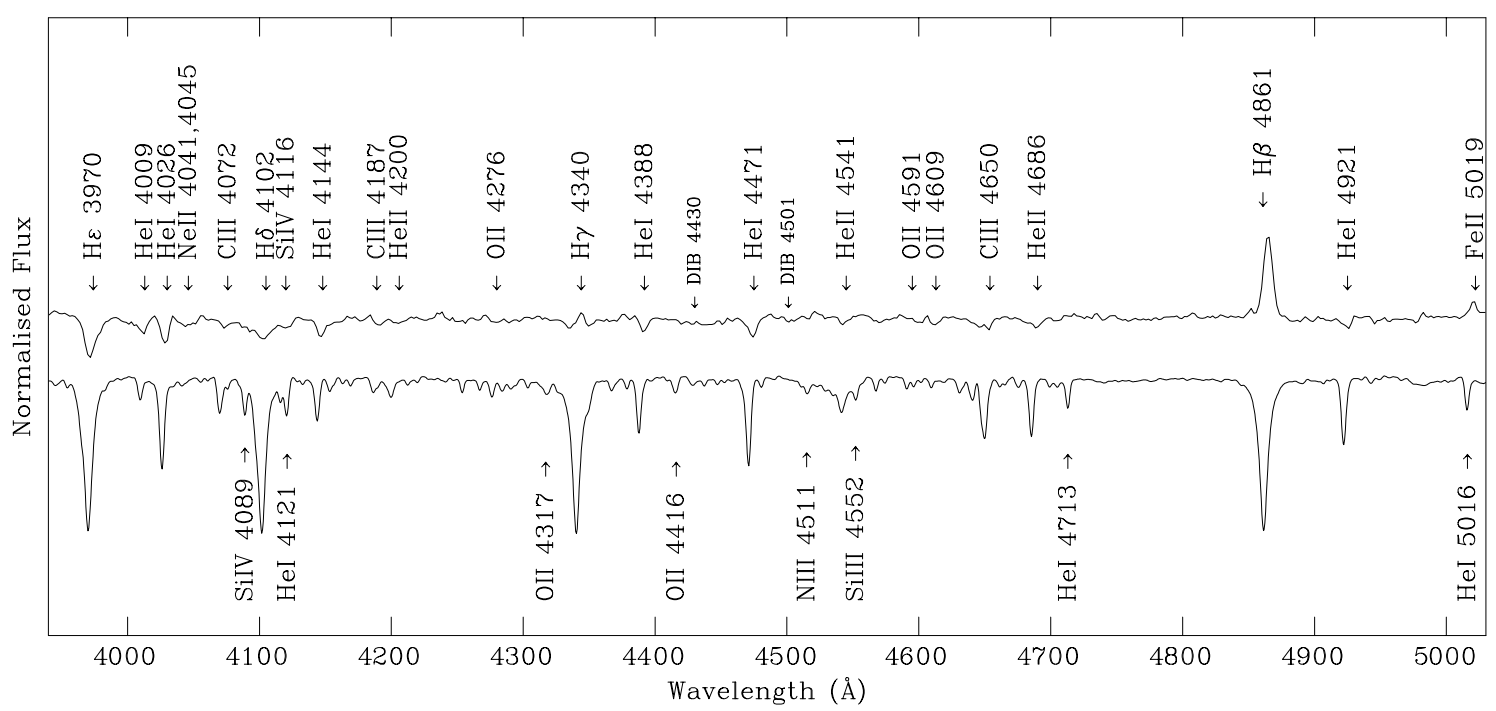

Fig. 3. Spectrum of the optical counterpart of RX J0052-7319 in the classification region compared to the B0V standard $\nu$ Ori. Note the broadness and shallowness of all features in RX J0052-7319. Some relatively prominent lines not seen in the spectrum of RX J0052-7319 are marked on the spectrum of $\nu$ Ori. Both spectra have been divided by a spline fit to the continuum for normalization and smoothed with a $\sigma=1.2$ Gaussian filter. The identification of $\mathrm{O}$ II lines is tentative, since they do not correspond well to the derived spectral type, and the features could be notches created by the presence of weak Fe II emission lines.

we can assume that the interstellar reddening takes a value $E_{\text {is }}(B-V)=0.08$ typical for the $\mathrm{SMC}$ and that the rest of the reddening is circumstellar, in which case $M_{V}=-4.4 \pm 0.15$. This value is compatible with a B0V star with $E_{\mathrm{cs}}(B-V)=0.3$, though we note that such circumstellar emission would be rather high. Even though the weakness of all the Si lines ( $\mathrm{Si}$ IV $\lambda 4089 \AA$ is hidden on the blue wing of $\mathrm{H} \delta$ ) would support a main sequence classification for a Galactic object, we note that the metal content of the SMC is much lower than that of the Milky Way and that even supergiants in the SMC can have metallic lines as weak as those of main-sequence objects in our Galaxy (Walborn 1983; see also Lennon 1997). As a consequence, we find that we cannot decide on the luminosity class of the star without knowing how much of the reddening is circumstellar. If it is a giant, the presence of weak He II $\lambda 4200 \AA$ would indicate spectral type O9.5IIIe, while B0Ve would be the spectral classification if it is on the main sequence.

\subsection{XTE J0111.2-7317}

The X-ray transient XTE J0111.2-7317 was discovered by the RXTE X-ray observatory in November 1998 (Chakrabarty et al. 1998a). Analysis of ASCA observation (Chakrabarty et al. 1998b; Yokogawa et al. 2000b) identified this source as as a $31 \mathrm{~s} \mathrm{X}$-ray pulsar with a flux in the $0.7 \div 10 \mathrm{keV}$ band of $3.6 \times 10^{-10} \mathrm{erg} \mathrm{s}^{-1} \mathrm{~cm}^{-2}$ and $\sim 45 \%$ pulsed fraction. The detection was also confirmed from the BATSE telescope on the CGRO satellite which detected the source in the hard $20 \div 50 \mathrm{keV}$ band with a flux ranging from 18 to $30 \mathrm{~m}$ Crab (Wilson \& Finger 1998).
The best X-ray position for XTE J0111.2-7317 was inferred from the ASCA observation carried out during the 1998 outburst (Yokogawa et al. 2000b). Moreover, we applied the new calibration for the restoration of the ASCA source position accuracy applying the algorithm described by Gotthelf et al. (2000). This yielded the following improved position of the source for the ASCA-SIS observation: $\mathrm{RA}=1^{\mathrm{h}} 11^{\mathrm{m}} 11.5 .5$, DEC $=-73^{\mathrm{o}} 16^{\prime} 44.6^{\prime \prime}$ (J2000), with an error radius of $15^{\prime \prime}$ (90\% confidence). As a reference, we also report the position as inferred in the analysis of the ASCA-GIS observation: RA = $1^{\mathrm{h}} 11^{\mathrm{m}} 12.3 \mathrm{~s} .5, \mathrm{DEC}=-73^{\circ} 16^{\prime} 47.6^{\prime \prime}$ (J2000), with an error radius of $25^{\prime \prime}$ (90\% confidence).

No ROSAT observations were able to detect this source. Summing up all the 6 available PSPC observations we are able to derive a $3 \sigma$ upper limit of $1.4 \times 10^{-3} \mathrm{c} \mathrm{s}^{-1}$ (0.1-2.4 keV, for a total exposure time of $\sim 90 \mathrm{ks})$, which translates to a luminosity limit of $\sim 3 \times 10^{34} \mathrm{erg} \mathrm{s}^{-1}$ in the $0.1 \div 2.4 \mathrm{keV}$ energy band for a Crab-like spectrum and a column density of $10^{21} \mathrm{~cm}^{2}$. These observations clearly indicate the transient nature of this source.

A Be star counterpart was proposed by Israel et al. (1999b) and subsequently confirmed by Coe et al. (2000) as a B0-B2 star with strong evidence for the presence of a surrounding nebula, possibly a SuperNova Remnant (SNR).

Images for this field were taken (see Fig. 4) revealing two bright stars within the $15^{\prime \prime}$ error circle. The fainter one $($ star B $)$ is a B-type star $(R=15.29 \pm 0.03, V-$ $R=+0.06 \pm 0.04)$, located at the coordinates (J2000, estimated uncertainty $\left.1^{\prime \prime}\right) \mathrm{RA}=01^{\mathrm{h}} 11^{\mathrm{m}} 08^{\mathrm{s}} 4$, DEC $=$ $-73^{\circ} 16^{\prime} 46^{\prime \prime}$ 


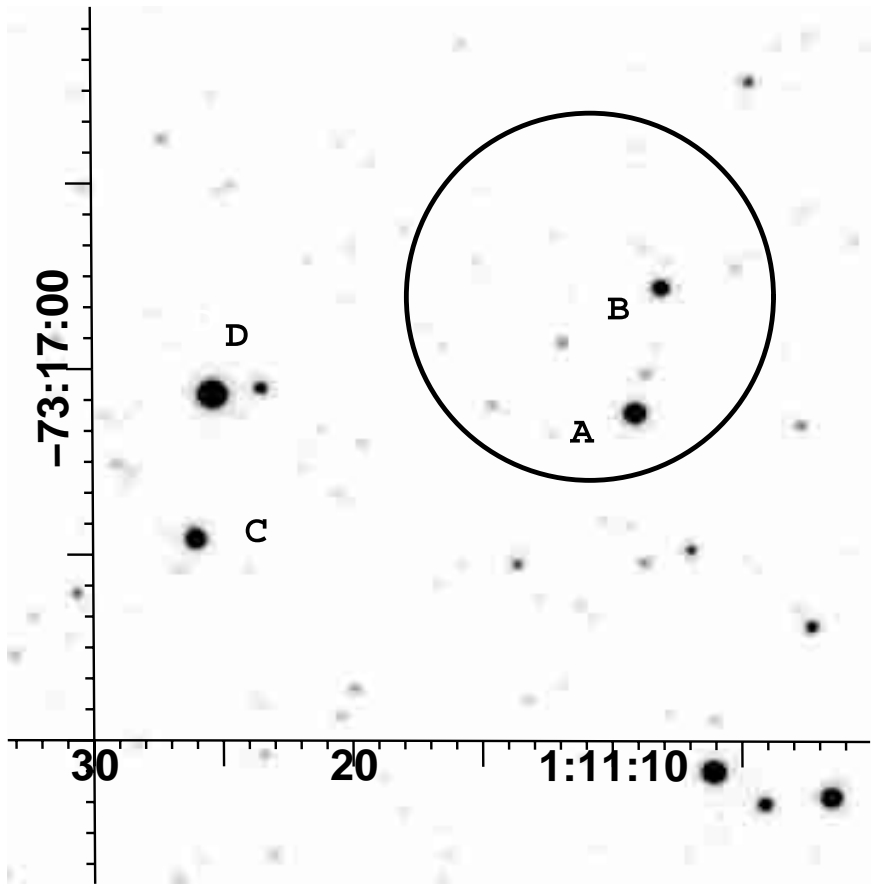

Fig. 4. 1.5 m ESO Danish telescope XTE J0111.2-7317 $R$ image. The refined ASCA-SIS X-ray error circle is also shown. The two brightest stars in the error circle are labeled A and $\mathrm{B}$; while the other two bright stars just outside the error circle are labeled as $\mathrm{C}$ and $\mathrm{D}$.

A $1,000 \mathrm{~s}$ spectrum taken with the $1.5 \mathrm{~m}$ Danish telescope (Table 1) revealed strong $\mathrm{H} \alpha$ and $\mathrm{H} \beta$ emission lines $(E W \sim-21$ and $-1.5 \AA$, respectively), indicating that this source is likely the counterpart of the $\mathrm{X}$-ray transient. The brighter star $(R=14.30 \pm 0.03)$ does not show any emission line. No other object brighter than $R \sim 17.3$ is found within the error circle. One more $\mathrm{B}-$ type $\operatorname{star}(R=14.55 \pm 0.03, V-R=+0.05 \pm 0.04)$, located at the coordinates (J2000, estimated uncertainty $1^{\prime \prime}$ ) $\mathrm{RA}=01^{\mathrm{h}} 11^{\mathrm{m}} 25 \mathrm{~s} .9, \mathrm{DEC}=-73^{\circ} 17^{\prime} 27^{\prime \prime}\left(1^{\prime}\right.$ away from the $\mathrm{X}$-ray position, star $\mathrm{C}$ ) also shows strong $\mathrm{H} \alpha$ and $\mathrm{H} \beta$ emission lines ( $E W=-36$ and $-3 \AA$, respectively).

In order to infer an accurate spectral classification of the selected objects we observed again the field and, on 1999, September 15th, we set the slit so that the bright Be star just outside the error circle (star C) was also included in the slit. This star can be seen on the Eastern edge of all the images in Coe et al. (2000) and in our Fig. 4. The orientation of the slit was therefore almost E-W. On 1999 November 1st, on the other hand, we set the slit so that the core of the dark nebulous region that can be seen in Fig. 2 of Coe et al. (2000) to the SW of star B was inside the slit.

The two spectra of star B in the classification region are displayed in Fig. 6. The spectrum from November 1st has a higher resolution, but the spectrum from September 15 th has rather higher $S / N$. Also displayed is the spectrum of star $\mathrm{C}$, which shows $\mathrm{H} \beta$ in emission and emission components in other Balmer series lines. The measured $\mathrm{H} \beta E W=-3.3 \AA$ is typical of Be stars with strong emission. This is confirmed by the red spectrum of the source (see Fig. 7), where $\mathrm{H} \alpha$ is strongly in emission $(E W=-40 \AA)$. Typically, Be stars with such strong emission in the Balmer series also display many weak emission lines corresponding to low-ionization metallic transitions (mainly Fe II). The emission spectrum of star C is clearly seen in Fig. 7.

The blue spectrum of star $\mathrm{C}$ shows weak He II lines, indicating a spectral type earlier than B0.5. The weakness of all metallic lines (and specifically the Si IV lines in the wings of $\mathrm{H} \delta$ ) suggests a main sequence object (though see previous section). The weak He II $\lambda \lambda 4200,4541 \AA$ indicate a spectral type O9.5-B0, while He II $\lambda 4686 \AA \lesssim$ He I $\lambda 4713 \AA$ favors the latter classification. Assuming a distance modulus $(m-M)=18.8$ (Coe et al. 2000), the measured $V=14.6$ implies a $M_{V}=$ -4.2 , consistent with a B0V star (Vacca et al. 1996). Considering an interstellar reddening $\approx 0.14$ (Coe et al. 2000 ), the source must actually have $M_{V}=-4.6$, which is slightly too high for a main sequence object. However, Be stars tend to be brighter than that corresponds to their spectral type due to the continuum emission from the circumstellar disc. Zorec \& Briot (1991) and Fabregat \& Torrejón (1998) give an average difference of $0.3 \mathrm{mag}$ between Be stars and main-sequence objects. Using the approximate relationship of Fabregat \& Reglero (1990), which is valid for isolated Be stars, an $\mathrm{H} \alpha E W=-40 \AA$ implies a circumstellar brightening $\Delta V \simeq 0.3$. Therefore star $\mathrm{C}$ has a spectral type (B0Ve) and spectral morphology typical of a counterpart to Be/X-ray binary.

The spectrum of star B, on the other hand, does not show obvious He II lines, indicating a later spectral type. The only photospheric features that could be visible in its spectrum and are not marked on the spectrum of star $\mathrm{C}$ are the $\mathrm{O}$ II blends at $\lambda \lambda 4146-53$ and $4185-90 \AA$, He I $\lambda 4169 \AA$ and perhaps N II $\lambda 4237-4242 \AA$. These are possible identifications for features that are visible in both spectra of the source. However, from the two spectra, it is clear that longwards of $\approx \lambda 4200 \AA$ the observed "continuum" actually consists of a forest of weak emission lines, that create apparent absorption features, such as the shallow band at $\sim \lambda 4600 \AA$ in the November spectrum, which is probably only a gap between emission components centered on Fe II $\lambda 4584 \AA$ and Fe II $\lambda 4629 \AA$.

If the weak feature seen at $\approx \lambda 4689 \AA$ in both spectra is real and can be identified with He II $\lambda 4686 \AA$ (with the radial velocity shift of $165 \mathrm{~km} \mathrm{~s}^{-1}$ measured by Coe et al. 2000), then the spectral type would be B0.5-B0.7. The presence of the strong O II/C III blend at $\approx \lambda 4650 \AA$ indicates that it cannot be later than B1 in any case.

Longwards of $\mathrm{H} \beta$ the FeII emission spectrum is very strong (see Fig. 7). Close to $\lambda 5000 \AA$, apart from Fe II $\lambda 5018 \AA$, two other lines, which are particularly strong in the November spectrum, can be seen. Their wavelengths suggest identification with the [O III] $\lambda \lambda 4959$ and $5007 \AA$ lines. Such lines are not generally seen on stellar spectra, but are typical of nebular emission. 

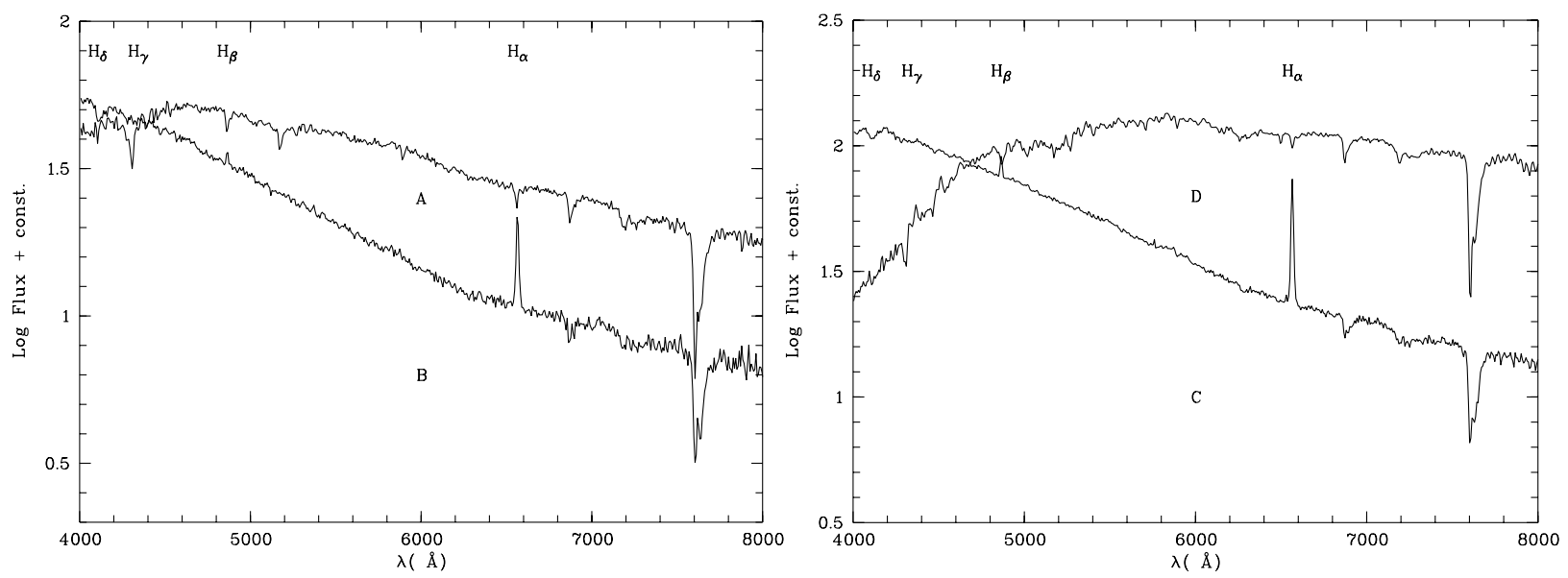

Fig. 5. 1.5 m ESO Danish telescope XTE J0111.2-7317 spectrum for objects A, B (left panel), C and D (right panel) in the $\mathrm{X}$-ray error circle. Position of the main hydrogen lines is also shown.

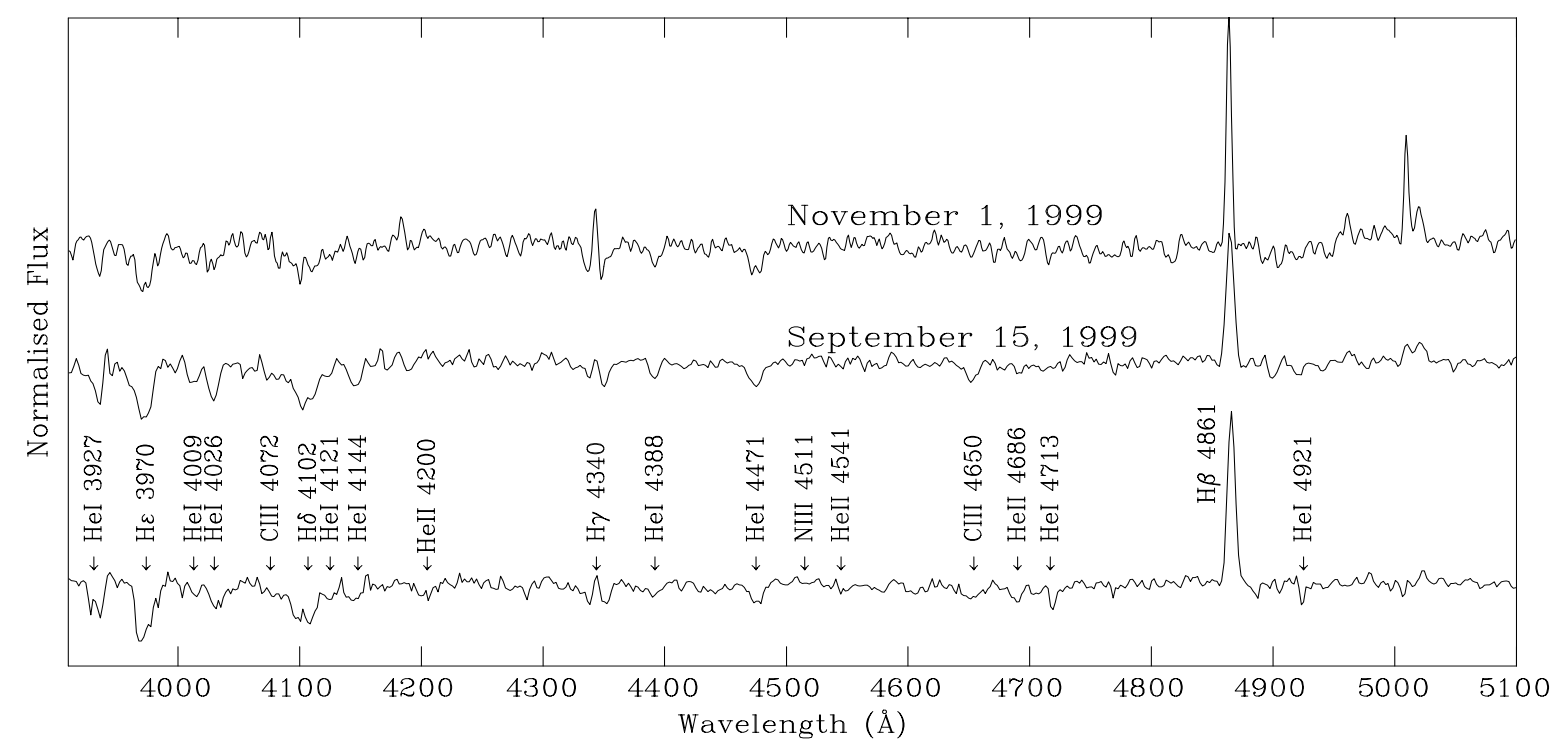

Fig. 6. The two blue spectra of the optical counterpart to XTE J0111.2-7317 (top) compared to that of the B0Ve star just outside the error circle (star C, bottom). The lines marked on the spectrum of star C are characteristic of the spectral type (compare with the spectroscopic standard in Fig. 3). All spectra have been divided by a spline fit to the continuum for normalization and smoothed with a $\sigma=1.2$ Gaussian filter. All identified features are present in at least two spectra.

Diffuse emission is actually apparent on the region immediately to the East of star B in our September image. When the sky spectrum from that area is extracted and a sky spectrum from a distant area is subtracted, strong emission lines corresponding to [O II] $\lambda 3727 \AA, \mathrm{H} \alpha, \mathrm{H} \beta$, $\mathrm{H}_{\gamma},[\mathrm{O}$ III] $\lambda \lambda 4959,5007 \AA$ and presumably [S II] $\lambda 6716 \AA$ can be seen.

In the image corresponding to the November observation, diffuse emission is present on the region immediately to the SouthWest of star B. [O II] $\lambda 3727 \AA, \mathrm{H} \beta$, and [O III] $\lambda \lambda 4959,5007 \AA$ are readily seen in the extracted sky spectrum.

Extracting several sky spectra around star B, we come to the conclusion that most of the nebular line emission comes from the immediate vicinity of the star and that it is strongest on the spatial position of the star. Therefore we conclude that a significant portion of the emission seen on star B comes from the nebulosity surrounding it and we are tempted to attribute (at least part of) the obvious differences between the September and November spectra to the different slit orientations.

However, it is clear from the detection of many permitted Fe II emission lines that star B is also intrinsically an emission line star, i.e., is surrounded by a circumstellar envelope. As a matter of fact, we believe that star B is also a Be star, since other possibilities seem to be ruled out because of the following points: (i) the detected forbidden lines are typical of high excitation nebulae and not of $\mathrm{B}[\mathrm{e}]$ stars. (ii) The presence of a compact companion precludes the possibility that star B is a Herbig Be star. (iii) The $E W \mathrm{~s}$ of $\mathrm{H} \alpha$ and $\mathrm{H} \beta$ are typical of Be stars and very low for any $\mathrm{B}[\mathrm{e}]$ star. (iv) If star $\mathrm{B}$ was a $\mathrm{B}[\mathrm{e}]$ star, it should show narrow emission lines corresponding to [Fe II]. 


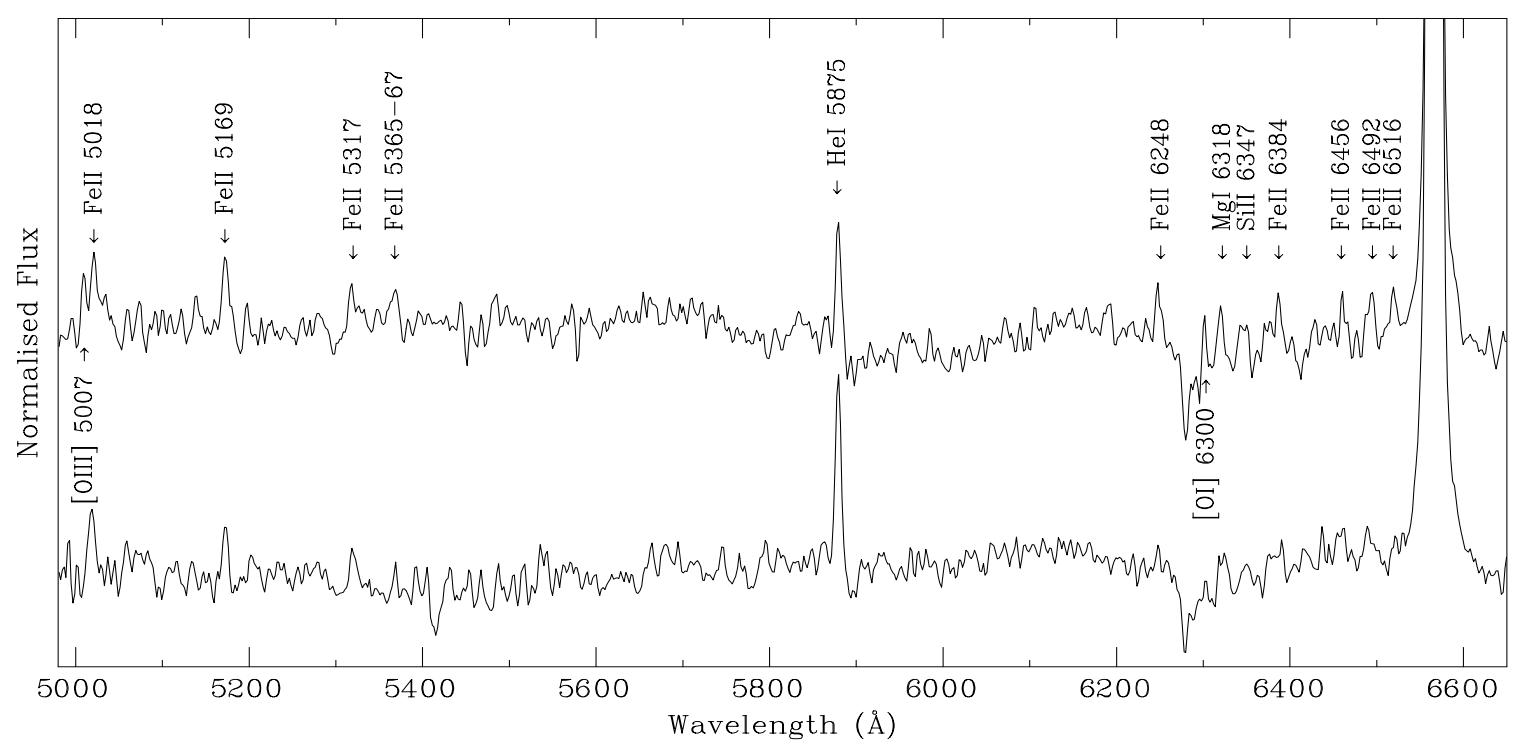

Fig. 7. Red spectra of the optical counterpart to XTE J0111.2-7317 (top) and the B0Ve star just outside the error circle (star $\mathrm{C}$, bottom). The many permitted emission lines on the spectrum of the optical counterpart to XTE J0111.2-7317 indicate that it is intrinsically a Be star, while the presence of weak forbidden lines must be due to emission from the surrounding nebula. Both spectra have been divided by a spline fit to the continuum for normalization and smoothed with a $\sigma=1.2$ Gaussian filter.

Table 3. Photometry for selected objects in the fields of RX J0052.1-7319 and XTE J0111.2-7317. The proposed counterparts are labeled by a "*". Errors are between $0.03 \div$ $0.05 \mathrm{mag}$.

\begin{tabular}{llcccl}
\hline Field & Star & $B$ & $V$ & $R$ & Spectral type \\
\hline RX J0052.1-7319 & A $^{*}$ & 14.73 & 14.62 & 14.54 & O9.5IIIe \\
& B & 15.82 & 15.92 & 16.05 & \\
XTE J0111.2-7317 & A & 15.32 & 14.65 & 14.30 & \\
& B $^{*}$ & 15.42 & 15.36 & 15.29 & B0.5-B1Ve \\
& C & 14.63 & 14.61 & 14.55 & B0Ve \\
& D & 15.05 & 13.67 & & \\
\hline
\end{tabular}

Moreover, the $E(B-V)=0.2-0.3$ derived by Coe et al. (2000) shows that the obscuration by the nebula is small. Therefore the luminosity of star B is far too low for a $\mathrm{B}[\mathrm{e}]$ star.

Therefore star B has a spectral type B0.5-B1Ve, compatible with the $M_{V}=-3.8$ derived by Coe et al. (2000) and it is the likely counterpart to XTE J0111.2-7317. Its association with the nebulosity that apparently surrounds it is almost certain, though further study is necessary in order to assess its nature.

We note that our value $(B-V)=0.05$ would imply an $E(B-V)=0.28$, consistent with the value derived by Coe et al. (2000) from Strömgren photometry (though hardly consistent with the value they derived from Johnson photometry, which has $(B-V)=-0.08$. However, the two Coe at al. measurements of the $V$ magnitude reported for this star show a spread that is much larger than the measurement errors, indicating variability.

\section{Conclusions}

We discuss the optical conterparts of two transient $\mathrm{X}$ ray pulsars in the SMC. In the case of RX J0052.1-7319, ROSAT HRI observations allowed us to derive an error circle of $\sim 3^{\prime \prime}$ radius $(90 \%)$. Accurate positioning can then be achieved with past HRI instruments if a boresight correction can be derived. Within the error circle only one relatively bright source is found with $V=14.6$ $(B-V=0.1)$. The object has been investigated photometrically and spectroscopically leading to classify it as a 09.5IIIe star (a classification as a B0Ve star is also possible, since the luminosity class depends on the adopted reddening). Medium resolution spectra show prominent $\mathrm{H} \alpha$ and $\mathrm{H} \beta$ emission lines, further strengthening the identification.

The position of XTE J0111.2-7317 is based on ASCA observations analyzed by the new calibration for the restoration of the ASCA source position accuracy described by Gotthelf et al. (2000). The analysis provided a $15^{\prime \prime}$ error box. Within it we found a relatively bright object $(V=15.4, B-V=0.06)$ which can be classified as a B0.5-B1Ve star. Also in this case we easily detect Balmer emission lines. A further bright B0Ve star was found outside the X-ray error circle. Coe et al. (2000) found evidence for the presence of a surrounding nebula, possibly a supernova remnant, around the likely counterpart of XTE J0111.2-7317.

Acknowledgements. SCo thanks the Rome Astronomical Observatory for the kind hospitality. During part of this work, IN was supported by an external ESA fellowship. This work is partially supported through CNAA, ASI and MURST grants. We also thank the anonymous referee for his quick reply and useful comments, suggestions and corrections that increased the readability of the paper. 


\section{References}

Campana, S., Lazzati, D., Panzera, M. R., et al. 1999, ApJ, 524,423

Chakrabarty, D., Levine, A. M., Clark, G. W., et al. 1998a, IAUC, 7048

Chakrabarty, D., Takeshima, T., Ozaki, M., Paul, B., \& Yokogawa, J. 1998b, IAUC, 7062

Coe, M. J., Haigh, N. J., \& Reig, P. 2000, MNRAS, 314, 290

Coe, M. J. 2000, Proceedings of IAU Colloq., 175, ed. M.A. Smith et al., ASP Conf. Ser., 214 [astro-ph/9911272]

Covino, S., Israel, G. L., Polcaro, V. F., et al. 2000, ASP Conf. Ser., 214, 681

Fabregat, J., \& Reglero, V. 1990, MNRAS, 247, 407

Fabregat, J., \& Torrejón, J. M. 1998, A\&A, 332, 643

Gotthelf, E. V., Ueda, Y., Fujimoto, R., Kii, T., \& Yamaoka, K. 2000, Apj, 543, 417

Haberl, F., Filipović, M. D., Pietsch, W., \& Kahabka, P. 2000, A\&A, 142, 41

Inoue, H., Koyama, K., \& Tanaka, Y. 1983, IAU Symp., 101, 535

Israel, G. L., Covino, S., Campana, S., et al. 2000a, MNRAS, $314, \mathrm{~L} 87$

Israel, G. L., Covino, S., Stella, L., et al. 1999a, ApJ, 518, L107

Israel, G. L., Covino, S., Polcaro, V. F., et al. 2000b, ASP Conf. Ser., 214, 739

Israel, G. L., Panzera, M. R., Campana, S., et al. 2000c, A\&A, 349, L1

Israel, G. L., Stella, L., Covino, S., Campana, S., \& Mereghetti, S. 1999b, IAUC, 7101

Kahabka, P. 1998, IAUC, 7082

Kahabka, P. 1999, IAUC, 7087

Kahabka, P. 2000, A\&A, 354, 999

Kahabka, P., \& Pietsch, W. 1996, A\&A, 312, 919

Kahabha, P., Pietsch, W., Filipovic, M. D., \& Haberl, F. 1999, A\&AS, 136, 81
Lamb, R. C., Prince, T. A., \& Finger, M. H. 1999, IAUC, 7081

Lazzati, D., Campana, S., Rosati, P., et al. 1999, ApJ, 524, 414

Lennon, D. J. 1997, A\&A, 317, 871

Liu, Q. Z., van Paradijs, J., \& van den Heuvel, E. P. J. 2000, A\&AS, 147, 25

Mereghetti, S. 2001, Vulcano workshop [astro-ph/0102017]

Mereghetti, S., Mignani, R. P., Covino, S., et al. 2001, MNRAS, 321,143

Nelson, R. W., Salpeter, E. E., \& Wasserman, I. 1993, ApJ, 418,874

Nelson, R. W., Wang, W., Salpeter, E. E., \& Wasserman, I. 1995, ApJ, 438, L99

Negueruela, I. 1998, A\&A, 338, 505

Negueruela, I., \& Okazaki, A. T. 2000, Proceedings of IAU Colloq. 175, ed. M.A. Smith et al., ASP Conf. Ser., 214, 713 [astro-ph/0011406]

Sasaki, M., Haberl, F., \& Pietsch, M. 2000, A\&A, 147, 75

Shortridge, K., Meyerdicks, H., Currie, M., et al. 1997, Starlink User Note 86.15 , R.A.L.

Stetson, P. B. 1987, PASP, 99, 191

Udalski, A., and the OGLE collaborations 1999, IAUC, 7105

Udalski, A. 2000, AcA, 50, 279

Vacca, W. D., Garmany, C. D., \& Shull, J. M. 1996, ApJ, 460, 914

Walborn, N. R. 1983, ApJ, 265, 716

Wang, Q., \& Xiaoyi, W. 1992, ApJS, 78, 391

Wegner, W. 1994, MNRAS, 270, 229

Wilson, C. A., \& Finger, M. H. 1998, IAUC 7048

Yokogawa, J., Biswajit, P., Masanobu, O., et al. 2000, ApJ, 539, 191

Yokogawa, J., Imanishi, K., Tsujimoto, M., et al. 2000, ApJS, 128,491

Zamanov, R., Reig, P., Martì, J., et al. 2001, A\&A, 367, 884

Zorec, J., \& Briot, D. 1991, A\&A, 245, 150 